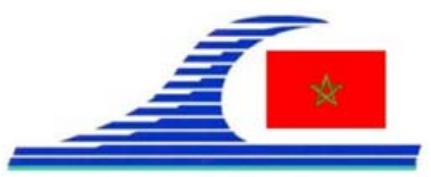

Conférence Méditerranéenne Côtière et Maritime EDITION 2, TANGER, MAROC (2011)

Coastal and Maritime Mediterranean Conference

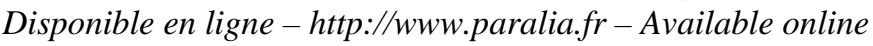

\title{
Érosion hydrique et transferts des matières vers les zones côtières méditerranéennes. Cas du bassin de l'oued Khmiss (Rif nord occidental. Maroc)
}

\author{
Ahmed RAISSOUNI ${ }^{1}$, Lamiae KHALI ISSA ${ }^{1}$, Rachid MOUSSADEK ${ }^{2}$, \\ Rachid MRABET ${ }^{3}$, Abdelkrim EL ARRIM ${ }^{1}$
}

1. Département des Sciences de la Terre. Faculté Des Sciences Et Techniques. BP 416 Tanger, Maroc.

ah_raissouni@hotmail.com

2. Laboratoire de cartographie, SIG et Télédétection. Unité de 1'Environnement et Ressources Naturelles. INRA. BP 415 RP Rabat, Maroc.

moussa.inra@gmail.com

3. Centre Régional des Recherches Agronomiques, INRA. 78 Avenue Sidi Mohamed Ben Abdellah, Tanger, Maroc.

mrabet@inra.org.ma

\section{Résumé :}

L'objectif de cette étude est de développer une méthodologie utilisant la télédétection et les systèmes d'information géographique (SIG) pour cartographier, d'une façon homogène, les risques d'érosion hydrique dans le bassin versant de l'oued Khmiss. La cartographie de l'intensité de l'aléa d'érosion et des différents types de fonctionnement érosif a été réalisée en utilisant le modèle universel de perte en sol (USLE) adapté selon les conditions marocaines. La superposition des couches d'information sur les précipitations, les sols, la topographie et le couvert végétal, permet d'obtenir une carte qui présente l'aléa moyen de l'érosion en t/ha/an par unité spatiale. La carte obtenue montre que le bassin est généralement caractérisé par une érosion relativement modérée avec un taux moyen d'érosion de $37 \mathrm{t} / \mathrm{ha} / \mathrm{an}$.

\section{Mots-clés :}

USLE - SIG - Érosion hydrique - Bassin Versant - Oued Khmiss - Maroc

\section{Introduction}

L'érosion hydrique est un phénomène naturel qui requiert actuellement beaucoup d'intérêt. Elle prend une part importante dans les plans de développement et de l'aménagement des pays, du fait de ses conséquences sur site (dégradation des terres agricoles en particulier) et hors site (pollution des eaux, coulées boueuses, envasement des barrages, etc.). C'est un facteur primordial à prendre en considération par les décideurs dans le domaine environnemental. Cet intérêt réside dans le fait que les impacts de ce phénomène aléatoire et récurrent se présentent dans plusieurs domaines. 
La connaissance de la Mer :

un vecteur du développement durable en Méditerranée

L'érosion hydrique est également la source principale des matières en suspension. Un transfert excessif de ces matières vers les zones littorales peut avoir des effets non désirables tels que l'aggradation côtière accompagnée d'une diminution de la bathymétrie et participe fortement au problème d'ensablement des ports. La nature des MES, leur concentration et les polluants éventuels qu'elles contiennent ou transportent influencent la qualité de l'eau et perturbent la vie aquatique. L'accumulation des sédiments réduit la profondeur disponible pour la navigation et accroit les risques d'inondation. Le volume sédimentaire excessif a également des conséquences sur la flore et la faune sous marines.

A la lumière de ces conséquences néfastes, le phénomène de l'érosion revêt un intérêt particulier pour le développement économique des pays.

Dans ce sens, la modélisation et la cartographie de l'érosion est devenue actuellement une nécessité pour les décideurs afin de pouvoir cibler les zones à risque majeur et chercher des solutions adéquates. L'intégration des modèles existants avec les nouvelles techniques de spatialisation comme la télédétection et les systèmes d'information géographique ont permis de minimiser les coûts financiers et gagner le temps de cartographie des risques et par conséquence, l'intervention ciblée et rapide.

Dans ce contexte, cette étude vise à utiliser une modèle universel de perte de sol (USLE) intégré sous un Système d'Information Géographique (SIG) afin de quantifier et de cartographier l'aléa de l'érosion hydrique au niveau du bassin de l'oued Khmiss.

\section{Site d'étude}

Le bassin versant de l'oued Khmiss, avec une superficie de $293 \mathrm{~km}^{2}$, est situé au Nord Ouest du Maroc, dans la partie supérieure du bassin versant de l'oued Martil. Le climat est de type méditerranéen, caractérisé par des pluviométries sous forme d'averses concentrées pendant les saisons hivernales. Géologiquement, le bassin appartient au domaine rifain. Vers l'est se concentre les formations calcaires de la dorsale où culminent les hautes altitudes $(736 \mathrm{~m})$, alors que vers l'ouest se trouvent les terrains des flyschs gréso-pélitiques reposant sur l'unité externe de Tanger, essentiellement marnoargileuse, qui affleurent au centre du bassin à côté des basses terrasses fluviales quaternaires. L'occupation du sol est caractérisée globalement par les zones agricoles qui couvrent la plus grande superficie, suivies par des forêts et de la végétation sclérophylle. Les terres nues et les zones urbaines occupent des zones limitées.

\section{Méthodologie}

La modélisation du processus de l'érosion hydrique dans la région d'étude a été effectuée en utilisant le modèle USLE (Universal Soil Loss Equation) de WISCHMEIER et SMITH (1978) puis modifié par RENARD et al. (1997). L'érosion (A), exprimée en $\mathrm{t} / \mathrm{ha} / \mathrm{an}$, est le produit de 5 facteurs : 
- Le facteur d'agressivité climatique $R$ (en $\mathrm{MJ} / \mathrm{mm} / \mathrm{ha} \mathrm{H}$ an) a été calculé en 26 stations réparties dans la région en utilisant la formule $R=\Sigma^{1}{ }_{12}\left(M R i^{2}\right) / A R$ de ARNOLDUS (1977), adaptée aux conditions marocaines car elle ne prend en considération que les précipitations moyennes mensuelles $(M R)$ et annuelles $(A R)$ sur 30 ans. Les résultats obtenus présentent des valeurs variant entre 87 et 113 .

- Le facteur d'érodibilité du sol $K$ (en t ha $\mathrm{H} / \mathrm{ha} \mathrm{MJ} \mathrm{mm}$ ) a été calculé à partir des résultats des analyses physico-chimiques effectuées sur 42 sédiments de surface répartis dans le bassin par la formule de MERZOUK (1985) à trois paramètres:

$$
K=311.63-4.48 *(S G \%+S \%)+613.4 * E C+6.45
$$

Avec $S G \%$ : pourcentage du sable grossier ; $S \%$ : pourcentage du sable total et $E C$ : conductivité électrique $(\mathrm{mS} / \mathrm{cm})$.

L'interpolation des résultats du facteur $K$, montre une répartition spatiale des classes comprise entre 0,37 et 0,85 .

- Le facteur topographique $L S$ tient compte à la fois de la longueur (en m) et de l'inclinaison de la pente $S$ (en \%). Il a été calculé directement sous logiciel ArcGIS à partir du MNT, de la carte des classes de pentes (en degré) et la carte de la direction des flux selon l'expression suivante (MITASOVA et al., site web) :

$\operatorname{Pow}([$ flowacc]*resolution/22.1, 0.4)*Pow(Sin([slope]*0.01745 )/0.09, 1.4)*1.4

Le facteur $L S$ varie généralement de 5 à plus de 55 , avec une valeur moyenne de 7,91 .

- Le facteur du couvert végétal $\mathrm{C}$ a des valeurs qui varient de 1 sur un sol nu à 0 sur les plaines d'eau. Les valeurs de C (KALMAN, 1967) ont été attribuées pour chaque type de végétation correspondant sur une carte obtenue à partir de la classification d'une image satellitaire Landsat TM5 (tableau 1).

- Le facteur des pratiques anti-érosives $\mathrm{P}$ est considéré égal à 1 dans tout le bassin puisque aucune pratique n'y est réalisée.

Tableau 1. Facteur $C$ en fonction de l'unité de végétation.

\begin{tabular}{ll}
\hline Unité de végétation & Facteur C \\
\hline Eaux & 0 \\
Forêts & 0,001 \\
Matorral claire & 0,01 \\
Matorral dense & 0,05 \\
Zone agricole & 0,1 \\
Sol nu & 1 \\
\hline
\end{tabular}

\section{Résultats et discussions}

La carte d'aléa d'érosion des sols (figure 1.5) résulte de la combinaison de l'ensemble des facteurs calculés et rastérisés sous le pas du pixel de base du MNT (30 m de 
La connaissance de la Mer :

un vecteur du développement durable en Méditerranée

résolution) (figures 1.1, 1.2, 1.3 et 1.4). Le résultat est une carte quantitative présentant des classes comprises entre 0 et $>200$, avec une perte moyenne de $37 \mathrm{t} / \mathrm{ha} / \mathrm{an}$.

La distribution inégale des zones à risque potentiel d'érosion dans le bassin résulte de la variabilité des caractéristiques des facteurs impliqués dans les processus d'érosion. Par le biais de comparaisons avec les cartes de différents facteurs ainsi que des données géologiques, la carte de risque présente une érosion faible au centre (zones à pentes très faibles), Nord Est (forêts denses) et Sud Ouest (végétations sclérophylles) du bassin, et une érosion moyenne au niveau de l'Ouest et le Sud Est (formations de flyschs sur des pentes relativement fortes). L'érosion très élevé n'occupe que des zones limitées et concentrées surtout à l'extrémité Est du bassin, zones où se trouvent des terres nues sur des pentes relativement fortes et un relief élevé.

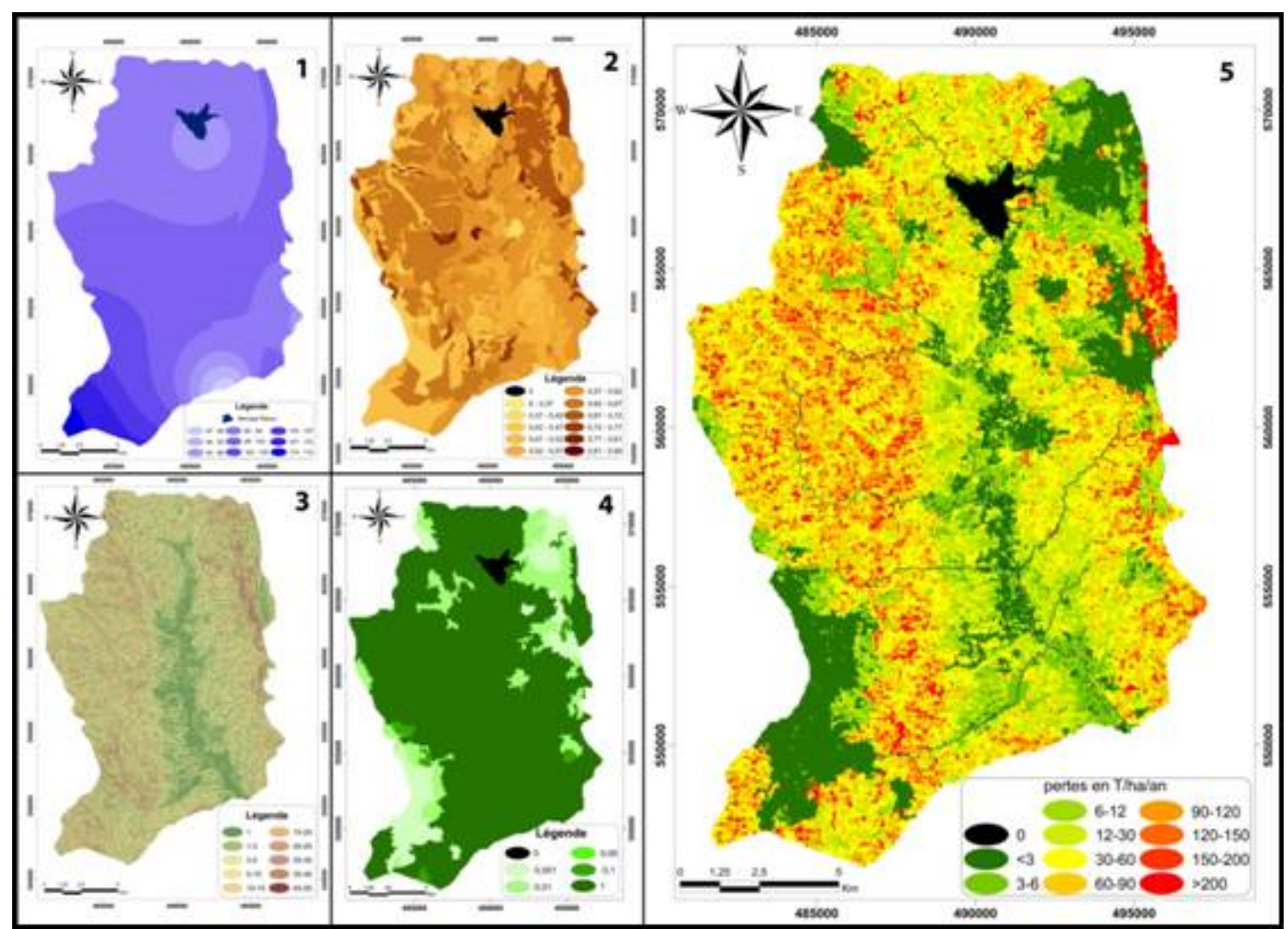

Figure 1. Cartes des facteurs de l'érosion à gauche $(1: R, 2: K, 3: L S$ et $4: C)$ et carte de risque potentiel d'érosion (5) en t/ha/an

LAOUINA et al. (1993), lors d'une étude sur les terrains du rif septentrional, a montré que la dégradation des terres varie de 5,85 t/ha/an sur un terrain entièrement boisé, à 18,5 sur un terrain à moitié défriché et cultivé puis à 94 sur un terrain totalement cultivé. Le taux d'érosion dans les zones rifaines fragiles est en moyenne de $17 \mathrm{t} / \mathrm{ha} / \mathrm{an}$ selon MOUKHCHANE et al. (1998) pouvant atteindre 49 t/ha/an selon ARNIL (1992). AÏT BRAHIM et al. (2003) ont calculé sur un autre sous bassin versant de l'oued 
Martil (bassin de Nakhla) une érosion moyenne de 38,7 t/ha/an. Ces différents résultats sont très proches de celles obtenues par cette étude ce qui montre la fiabilité relative du modèle appliqué.

D'autre part, une étude des quantités de matières en suspension au niveau de l'embouchure de l'oued Martil a donné, hors de la saison humide, des valeurs de $760 \mathrm{mg} / 1$ en 2010 et de $800 \mathrm{mg} / \mathrm{l}$ en 2011. Pour un débit moyen de l'ordre de 13,85 $\mathrm{m}^{3} / \mathrm{s}$, ces valeurs correspondent respectivement à 311575 et $327974 \mathrm{t} / \mathrm{an}$. Ces valeurs sont très proches de celles obtenues par d'autres auteurs sur le même secteur ou des secteurs proches: EL OUADINI et al. (2000) ont obtenu des valeurs de 0,2 à 11g/l. Quand à MAATOUK et al. (2003), ils ont attribué des valeurs atteignant 1 million t/an pour l'oued Martil et une valeur de 8,15 Mt/an pour tous les oueds débouchant dans la Méditerranée occidentale marocaine.

La distribution inégale des zones à risque potentielle d'érosion dans le bassin résulte de la variabilité des caractéristiques des facteurs impliqués dans le processus et le modèle utilisé. Tout d'abord, l'USLE donne seulement une estimation annuelle générale de la perte en sol attendue sur le long terme. Elle prédit seulement l'érosion diffuse et en rigoles : le ravinement n'est pas pris en compte. De plus, quelques facteurs importants influençant l'érosion du sol sont négligés, tel l'effet des pierres et des fragments de roches. Le cas d'estimation du facteur $R$, basée sur une relation approximée qui ne prend pas en considération les intensités des averses en $30 \mathrm{~min}$ et avec l'extrapolation des valeurs obtenues à l'ensemble du bassin, reste assez inapproprié à cause de la grande variété de micro-climats dans la région.

Malgré ces déficiences, les méthodes esquissées dans ce projet ont pu donner une information approuvable sur le risque d'érosion. Une estimation plus exacte peut être accomplie pour ces zones en utilisant des données plus détaillées, des modèles d'érosion plus sophistiqués et des études de terrain plus élargies.

\section{Conclusion}

La présente étude contribue à l'analyse et la quantification de l'érosion hydrique d'un bassin versant dans le Rif marocain. Le résultat montre l'intérêt de l'utilisation de la télédétection et des SIG dans l'évaluation de la vulnérabilité à l'érosion. Cette méthode de cartographie de risque érosif, reste un outil de gestion intéressant et un modèle assez fiable qui, avec des mises à jour continuelles et une amélioration des données sources, peut être un outil important pour l'évaluation spatio-temporelle du phénomène. La carte de risque d'érosion élaborée pourrait constituer un document de base pour tout aménagement proposé et permet aux décideurs de mieux cibler les zones prioritaires et de mieux planifier leurs stratégies d'intervention. 
La connaissance de la Mer :

un vecteur du développement durable en Méditerranée

\section{Références}

AÏT BRAHIM L., SOSSEY ALAOUI F., SITERI H., TAHRI M. (2003). Quantification des pertes en sols dans le bassin-versant Nakhla (Rif septentrional). Sécheresse, Vol. $14, \mathrm{n}^{\circ} 2$, pp 101-106.

ARNIL M. (1992). Recherche des zones sources d'envasement de la retenue du barrage M.B.A. El Khettabi (Maroc). Thèse 3ème cycle, Univ. Mohammed V, Rabat, 209 p.

ARNOLDUS H.M.J. (1977). Prédiction des pertes en terres par érosion en nappe et en griffe. Aménagement des bassins versants. Rome : FAO, pp 121-149.

EL OUADINI M., AIT BRAHIM L., MOUKHCHANE M., BENJBARA A., NACHITE D. (2000). Erosion dans le bassin versant de MARTIL, Maroc NO. Bull. Réseau Erosion 20, IRD, Montpellier, France, pp 78-86.

KALMAN R. (1967). Le facteur climatique de l'érosion dans le bassin du Sébou. Rabat : Ministère de l'Agriculture, $40 \mathrm{p}$.

LAOUINA A., CHAKER M., NACIRI R., NAFAA R. (1993). L'érosion anthropique en pays méditerranéen, le cas du Maroc septentrional. Bulletin de l'association de géographes français, Géographies, $\mathrm{n}^{\circ}$ 5, pp 384-398

MAATOUK M., EL ARRIM A., WAHBI M., EL MOUMNI B., BENMOUSSA A., PROBST J.L. (2003). Quantification des flux de matières en suspension à l'exutoire des bassins versants méditerranéens marocains. 1er colloque des Géosciences de l'environnement, Kénitra.

MERZOUK A. (1985) Relative Erodability of Nine Moroccan Soils as Related to Physical, Chemical and Minerlogic Properties. Ph D. thesis, University of Minnesota, $283 \mathrm{p}$.

MITASOVA H., BROWN W.M., HOHMANN M., WARREN S. (site web). Using Soil Erosion Modeling for Improved Conservation Planning: A GIS-based Tutorial. Geographic Modeling Systems Lab, University of Illinois at Urbana-Champaign. [http://skagit. meas.ncsu.edu/ helena/gmslab/reports/CerlErosionTutorial/denix/default.htm ]

RENARD K.G., FOSTER G.R., WEESIES G.A., MCCOOL D.K., YODER D.C., eds. (1997). Predicting soil erosion by water: a guide to conservation planning with the Revised Universal Soil Lass Equation (RUSLE). Agr. Handbook. Washington (DC) : USDA/US Government Printing Office, $407 \mathrm{p}$.

WISCHMEIER W.H., SMITH D.D. (1978). Predicting Rainfall Erosion Losses, a Guide to Conservation Planning. Agr. Handbook, $\mathrm{n}^{\circ}$ 537. Washington (DC): USDA, $58 \mathrm{p}$. 